\title{
Variant and invariant states for chemical reaction systems
}

\author{
D. Rodrigues, S. Srinivasan, J. Billeter, D. Bonvin* \\ Laboratoire d'Automatique, Ecole Polytechnique Fédérale de Lausanne, CH-1015 Lausanne, Switzerland
}

\section{A R T I C L E I N F O}

\section{Article history:}

Received 8 July 2014

Received in revised form 14 October 2014

Accepted 21 October 2014

Available online 11 November 2014

\section{Keywords:}

Chemical reaction systems

State decoupling

Reaction variants

Reaction invariants

Reaction extents

\begin{abstract}
A B S T R A C T
Models of chemical reaction systems can be quite complex as they typically include information regarding the reactions, the inlet and outlet flows, the transfer of species between phases and the transfer of heat. This paper builds on the concept of reaction variants/invariants and proposes a linear transformation that allows viewing a complex nonlinear chemical reaction system via decoupled dynamic variables, each one associated with a particular phenomenon such as a single chemical reaction, a specific mass transfer or heat transfer. Three aspects are discussed, namely, (i) the decoupling of reactions and transport phenomena in open non-isothermal both homogeneous and heterogeneous reactors, (ii) the decoupling of spatially distributed reaction systems such as tubular reactors, and (iii) the potential use of the decoupling transformation for the analysis of complex reaction systems, in particular in the absence of a kinetic model.
\end{abstract}

(c) 2014 Elsevier Ltd. All rights reserved.

\section{Introduction}

The (bio)chemical industry utilizes reaction processes to convert raw materials into desired products that include polymers, organic chemicals, vitamins, vaccines and drugs. If these processes involve chemical reactions, they also deal with (i) material exchange via inlet/outlet flows, mass transfers, convection, diffusion, and (ii) energy exchange via heating and cooling. Hence, modeling these phenomena is essential for improved process understanding, design and operation.

Models of chemical reaction processes are typically firstprinciples models that describe the state evolution (the mass, the concentrations, the temperature) by means of balance equations of differential nature (e.g. continuity equation, molar balances, heat balances) and constitutive equations of algebraic nature (e.g. equilibrium relationships, rate expressions). These models usually include information regarding the underlying reactions (e.g. stoichiometries, reaction kinetics, heats of reaction), the transfers of mass within and between phases, and the operating mode of the reactor (e.g. initial conditions, external exchange terms, operating constraints). A reliable description of reaction kinetics and transport phenomena represents the main challenge in building first-principles models for chemical reaction systems. In practice, such a description is constructed from experimental data

\footnotetext{
* Corresponding author. Tel.: +41 21693 3843; fax: +41 216932574 .

E-mail address: dominique.bonvin@epfl.ch (D. Bonvin).
}

collected both in the laboratory and during production (Marquardt, 2008).

The presence of all these phenomena, and in particular their interactions, complicates the analysis and operation of chemical reactors. The analysis would be much simpler if one could somehow separate the effect of the various phenomena and investigate each phenomenon individually. Ideally, one would like to have true variants, whereby each variant depends only on one phenomenon, and invariants that are identically zero and can be discarded. Note that some of the state variables are often redundant, as there are typically more states (balance equations) than there are independent source of variability (reactions, exchange terms). Hence, one would like to have a systematic way of discarding the redundant state variables, thereby reducing the dimensionality of the model.

Asbjørnsen and co-workers (Asbjornsen and Fjeld, 1970; Asbjornsen, 1972; Fjeld et al., 1974) introduced the concepts of reaction variants and reaction invariants and used them for reactor modeling and control. However, the reaction variants proposed in the literature encompass more than the reaction contributions since they are also affected by the inlet and outlet flows. Hence, Friedly $(1991,1996)$ proposed to compute the extents of "equivalent batch reactions", associating the remainder to transport processes. For open homogeneous reaction systems, Srinivasan et al. (1998) developed a nonlinear transformation of the numbers of moles to reaction variants, flow variants, and reaction and flow invariants, thereby separating the effects of reactions and flows. Later, the same authors (Amrhein et al., 2010) refined that transformation to make it linear (at the price of losing the one-to-one 
property) and therefore more easily interpretable and applicable. They also showed that, for a reactor with an outlet flow, the concept of vessel extent is most useful, as it represents the amount of material associated with a given process (reaction, exchange) that is still in the vessel. Bhatt et al. (2010) extended that concept to heterogeneous $\mathrm{G}-\mathrm{L}$ reaction systems for the case of no reaction and no accumulation in the film, the result being decoupled vessel extents of reaction, mass transfer, inlet and outlet, as well as true invariants that are identically equal to zero. An extension regarding the incorporation of calorimetric measurements into the extent-based identification framework has been proposed recently by Srinivasan et al. (2012).

Various implications of reaction variants/invariants have been studied in the literature. For example, Srinivasan et al. (1998) discussed the implications of reaction and flow variants/invariants for control-related tasks such as model reduction, state accessibility, state reconstruction and feedback linearizability. On the one hand, control laws using reaction variants have been proposed for continuous stirred-tank reactors (Hammarstrom, 1979; Waller and Mäkilä, 1981; Dochain et al., 2009; Favache and Dochain, 2009). The concept of extent of reaction is very useful to describe the dynamic behavior of a chemical reaction since a reaction rate is simply the derivative of the corresponding extent of reaction. Bonvin and Rippin (1990) used batch extents of reaction to identify stoichiometric models without the knowledge of reaction kinetics. Reaction extents have been used extensively for the kinetic identification of both homogeneous and $\mathrm{G}-\mathrm{L}$ reaction systems using either concentration (Bhatt et al., 2012) or spectroscopic (Billeter et al., 2013) measurements.

On the other hand, the fact that reaction invariants are independent of reaction progress has also been exploited for process analysis, design and control. For example, reaction invariants have been used to study the state controllability and observability of continuous stirred-tank reactors (Fjeld et al., 1974; Bastin and Lévine, 1993). Reaction invariants have also been used to automate the task of formulating mole balance equations for the non-reacting part (such as mixing and splitting operations) of complex processes, thereby helping determine the number of degrees of freedom for process synthesis (Gadewar et al., 2002). Furthermore, Waller and Mäkilä (1981) demonstrated the use of reaction invariants to control $\mathrm{pH}$, assuming that the equilibrium reactions are very fast. Grüner et al. (2006) showed that, through the use of reaction invariants, the dynamic behavior of reaction-separation processes with fast (equilibrium) reactions resembles the dynamic behavior of corresponding non-reactive systems in a reduced set of transformed variables. Aggarwal et al. (2011) considered multi-phase reactors operating at thermodynamic equilibrium and were able to use the concept of reaction invariants, which they labeled invariant inventories, to reduce the order of the dynamic model and use it for control.

This paper addresses the computation of variant and invariant states for reaction systems. It presents both existing approaches and novel techniques on a unified basis, which eases comparison. One will see that, not only reaction-variant states can be separated from reaction-invariant states, but a much finer separation can be achieved. The objective of this paper is therefore to sketch new avenues that could possibly lead to improved analysis, estimation, control and optimization of reaction systems.

The paper is organized as follows. Section 2 presents a novel way of computing the vessel extents of reaction and flow for open non-isothermal homogeneous reactors. The approach is extended to models that include a heat balance in Section 3 and to fluid-fluid reaction systems in Section 4, while Section 5 generalizes the transformation to distributed tubular reactors. The applicability of the decoupling transformation is discussed in Section 6, while Section 7 concludes the paper.

\section{Homogeneous reaction systems}

This section presents the computation of the extents of reaction and flow for a homogeneous reaction system with several inlets and one outlet. Although the computed extents are exactly the same as those in Amrhein et al. (2010), the computational approach is different and provides considerable insight in the transformation. This insight will help extend the transformation to more complex reaction systems in Sections 3-5.

\subsection{Mole balance equations}

Let us consider a general open non-isothermal homogeneous reactor. The mole balance equations for a reaction system involving $S$ species, $R$ reactions, $p$ inlet streams, and one outlet stream can be written as follows:

$\dot{\mathbf{n}}(t)=\mathbf{N}^{\mathrm{T}} \mathbf{r}_{v}(t)+\mathbf{W}_{i n} \mathbf{u}_{i n}(t)-\omega(t) \mathbf{n}(t), \quad \mathbf{n}(0)=\mathbf{n}_{0}$,

with

$\mathbf{r}_{v}(t):=V(t) \mathbf{r}(t)$

$\omega(t):=\frac{u_{\text {out }}(t)}{m(t)}$,

where $\mathbf{n}$ is the $S$-dimensional vector of numbers of moles, $\mathbf{r}$ the $R$ dimensional reaction rate vector, $\mathbf{u}_{\text {in }}$ the $p$-dimensional inlet mass flowrate vector, $u_{\text {out }}$ the outlet mass flowrate, $V$ and $m$ the volume and the mass of the reaction mixture. $\mathbf{N}$ is the $R \times S$ stoichiometric matrix, $\mathbf{W}_{i n}=\mathbf{M}_{w}^{-1} \check{\mathbf{W}}_{\text {in }}$ the $S \times p$ inlet-composition matrix, $\mathbf{M}_{w}$ the S-dimensional diagonal matrix of molecular weights, $\check{\mathbf{W}}_{i n}=$ $\left[\check{\mathbf{w}}_{i n}^{1} \ldots \check{\mathbf{w}}_{i n}^{p}\right]$ with $\check{\mathbf{w}}_{i n}^{j}$ being the $S$-dimensional vector of weight fractions of the $j$ th inlet flow, and $\mathbf{n}_{0}$ the $S$-dimensional vector of initial numbers of moles. Note that $\omega(t)$ corresponds to the inverse of the reactor residence time.

The mole balance Eq. (1a) holds independently of the operating conditions since the reaction rates are simply modeled as the unknown time signals $\mathbf{r}_{v}(t)$. The operating conditions such as the concentrations $\mathbf{c}(t)$ and the temperature $T(t)$ affect the reaction rates through the relations $\mathbf{r}_{v}(t)=V(t) \mathbf{r}(\mathbf{c}(t), T(t))$, but these dependencies are not needed at the level of Eq. (1a). If needed, the concentrations can be computed as $\mathbf{c}(t)=\mathbf{n}(t) / V(t)$, while the temperature can be described by a heat balance as shown in Section 3 . Note that the signals $\mathbf{r}_{v}(t)$ represent endogenous inputs.

The flowrates $\mathbf{u}_{\text {in }}(t)$ and $u_{\text {out }}(t)$ are considered as independent (input) variables in Eq. (1a). The way these variables are adjusted depends on the particular experimental situation; for example, some elements of $\mathbf{u}_{\text {in }}$ can be adjusted to control the temperature in a semi-batch reactor, or $u_{\text {out }}$ is a function of the inlet flows in a constant-mass reactor. The continuity equation (or total mass balance) is given by:

$$
\dot{m}(t)=\mathbf{1}_{p}^{\mathrm{T}} \mathbf{u}_{\text {in }}(t)-u_{\text {out }}(t), \quad m(0)=m_{0},
$$

where $\mathbf{1}_{p}$ is the $p$-dimensional vector filled with ones and $m_{0}$ the initial mass. Note that the mass $m(t)$ can also be computed from the numbers of moles $\mathbf{n}(t)$ as

$m(t)=\mathbf{1}_{S}^{\mathrm{T}} \mathbf{M}_{w} \mathbf{n}(t)$,

which indicates that Eqs. (1a) and (2) are in fact linearly dependent. Hence, the continuity equation is not needed per se, but it is often useful to express the mass as a function of the flows rather than the numbers of moles. The volume $V(t)$ can be inferred from the mass and knowledge of the density $\rho$ as $V(t)=m(t) / \rho(\mathbf{c}(t), T(t))$.

The analysis that follows will use intensively the following four integer numbers: 
- $S$ : the total number of species (reacting or not) in the reactor,

- $R$ : the total number of independent reactions,

- $p$ : the total number of independent inlet streams,

- $q$ : the number of invariant (redundant) states (introduced later).

\subsection{Reaction variants/invariants in the literature}

The concept of reaction variants/invariants as introduced by Asbjornsen and Fjeld (1970), Asbjornsen (1972), Fjeld et al. (1974) is briefly reviewed next. The idea is to use the stoichiometric matrix to construct a linear transformation of the states $\mathbf{n}$ to the reaction-variant states $\mathbf{y}_{r}$ and the reaction-invariant states $\mathbf{y}_{i v}$. This transformation $\mathcal{T}$ involves the stoichiometric matrix $\mathbf{N}$ and its null space of dimension $q=S-R$ described by the $S \times q$ matrix $\mathbf{P}$, that is $\mathbf{N} \mathbf{P}=\mathbf{0}_{R \times q}$ :

$$
\left[\begin{array}{l}
\mathbf{y}_{r}(t) \\
\mathbf{y}_{i v}(t)
\end{array}\right]=\mathcal{T} \mathbf{n}(t):=\left[\begin{array}{c}
\mathbf{R} \\
\mathbf{Q}
\end{array}\right] \mathbf{n}(t) \quad \mathcal{T}=\left[\begin{array}{ll}
\mathbf{N}^{\mathrm{T}} & \mathbf{P}
\end{array}\right]^{-1}
$$

It follows from $\mathbf{N} \mathbf{P}=\mathbf{0}_{R \times q}$ and $\quad \mathcal{T T}^{-1}=\left[\begin{array}{c}\mathbf{R} \\ \mathbf{Q}\end{array}\right]\left[\begin{array}{ll}\mathbf{N}^{\mathrm{T}} & \mathbf{P}\end{array}\right]=$ $\left[\begin{array}{ll}\mathbf{I}_{R} & \mathbf{0} \\ \mathbf{0} & \mathbf{I}_{q}\end{array}\right]$ that $\mathbf{R}=\mathbf{N}^{\mathrm{T}^{+}}$and $\mathbf{Q}=\mathbf{P}^{+}$, where $\mathbf{N}^{\mathrm{T}^{+}}$and $\mathbf{P}^{+}$represent the Moore-Penrose pseudo-inverse of $\mathbf{N}^{\mathrm{T}}$ and $\mathbf{P}$, respectively. ${ }^{1}$

The resulting dynamical system contains the $R$ state variables $\mathbf{y}_{r}$ that depend on the reactions and the $q$ state variables $\mathbf{y}_{i v}$ that do not:

$$
\begin{aligned}
\dot{\mathbf{y}}_{r}(t) & =\mathbf{r}_{v}(t)+\mathbf{N}^{\mathrm{T}^{+}} \mathbf{W}_{i n} \mathbf{u}_{i n}(t)-\omega(t) \mathbf{y}_{r}(t) & \mathbf{y}_{r}(0) & =\mathbf{N}^{\mathrm{T}^{+}} \mathbf{n}_{0} \\
\dot{\mathbf{y}}_{i v}(t) & =\mathbf{P}^{+} \mathbf{W}_{i n} \mathbf{u}_{i n}(t)-\omega(t) \mathbf{y}_{i v}(t) & \mathbf{y}_{i v}(0) & =\mathbf{P}^{+} \mathbf{n}_{0} .
\end{aligned}
$$

More specifically, one sees that the reaction variants are decoupled with respect to the reaction rates, that is, $y_{r, i}(t)$ depends on $r_{v, i}(t)$ but not on the other reaction rates:

$$
\begin{array}{r}
\dot{y}_{r, i}(t)=r_{v, i}(t)+\left(\mathbf{N}^{\mathrm{T}^{+}} \mathbf{W}_{i n}\right)_{i} \mathbf{u}_{i n}(t)-\omega(t) y_{r, i}(t) \\
y_{r, i}(0)=\left(\mathbf{N}^{\mathrm{T}^{+}}\right)_{i} \mathbf{n}_{0} \quad i=1, \ldots, R,
\end{array}
$$

where $(.)_{i}$ represents the $i$ th row of the matrix (.).

However, note that

- $\mathbf{y}_{r}$ are reaction and flow variants, and not solely reaction variants,

- $\mathbf{y}_{i v}$ are reaction invariants but flow variants, hence not truly invariants,

- $\mathbf{y}_{r}$ are pure reaction variants and $\mathbf{y}_{i v}$ are true invariants only for batch reactors, that is, for $\mathbf{u}_{i n}(t)=\mathbf{0}_{p}$ and $\omega(t)=0$, for which one can write:

$$
\dot{\mathbf{y}}_{r}(t)=\mathbf{r}_{v}(t) \quad \mathbf{y}_{r}(0)=\mathbf{N}^{\mathrm{T}^{+}} \mathbf{n}_{0} \quad \mathbf{y}_{i v}(t)=\mathbf{P}^{+} \mathbf{n}_{0},
$$

- $\mathbf{y}_{r}$ and $\mathbf{y}_{i v}$ are abstract mathematical quantities with no direct physical meaning.

Hence, the question arises whether it is possible to compute true reaction variants and true invariants for open reactors, thereby removing the effect of the inlet and outlet flows. The next section will show that this is possible with the concept of vessel extents.

\footnotetext{
1 Note that both $\mathbf{P}^{\mathrm{T}}$ and $\mathbf{P}^{+}$span the $q$-dimensional null space of the stoichiometric matrix. Note also that the stoichiometric matrix $\mathbf{N}$ satisfies the relationship $\mathbf{M} \mathbf{N}^{\mathrm{T}}=\mathbf{0}_{A \times R}$, where $\mathbf{M}$ is the $A \times S$ atomic matrix indicating the number of each atom type in the various species (Schneider and Reklaitis, 1975; Bonvin and Rippin, 1990). The maximum number of independent reactions is given by $R_{\max }=S-\operatorname{rank}(\mathbf{M}) \geq R$. (Gadewar et al., 2001) It follows that $q=S-R \geq S-R_{\max }=\operatorname{rank}(\mathbf{M})$.
}

\subsection{Vessel extents and true invariants}

For the sake of deriving the transformation, we will express the initial conditions as a unit impulse at time $0 .^{2}$ As a result, the balance Eq. (1a) can be written with zero initial conditions: ${ }^{3}$

$$
\dot{\mathbf{n}}(t)=\mathbf{N}^{\mathrm{T}} \mathbf{r}_{v}(t)+\mathbf{W}_{i n} \mathbf{u}_{i n}(t)+\mathbf{n}_{0} \delta(t)-\omega(t) \mathbf{n}(t), \quad \mathbf{n}(0)=\mathbf{0}_{S}
$$

Eq. (8) clearly explicits the fact that the initial conditions can be considered as a rate process (the process of instantaneously loading the reactor) that can be put on the same footing as the other rate processes. The right-hand side of Eq. (8) has four contributions that indicate the effects of the reactions, the inlets, the initial conditions, and the outlet, respectively. Note that the first three contributions have a particular structure, namely, they are expressed as the product of a constant term and a rate signal. This particular structure will be exploited for decoupling next.

\subsubsection{Decoupling transformation}

We look for a full-rank linear transformation that transforms $\mathbf{n}(t)$ into the three decoupled parts $\mathbf{x}_{r}(t), \mathbf{x}_{i n}(t)$ and $x_{i c}(t)$ and an orthogonal remaining part $\mathbf{x}_{i v}(t)$. The first three parts correspond to the reactions, the inlets and the initial conditions, while the remaining part is invariant as will be seen below. The linear transformation $\mathcal{T}$ reads:

$\left[\begin{array}{c}\mathbf{x}_{r}(t) \\ \mathbf{x}_{i n}(t) \\ x_{i c}(t) \\ \mathbf{x}_{i v}(t)\end{array}\right]=\mathcal{T} \mathbf{n}(t):=\left[\begin{array}{c}\mathbf{R} \\ \mathbf{F} \\ \mathbf{i}^{\mathrm{T}} \\ \mathbf{Q}\end{array}\right] \mathbf{n}(t)$,

and brings the dynamic model (8) to the following decoupled form:

$$
\begin{array}{ll}
\dot{\mathbf{x}}_{r}(t)=\underbrace{\mathbf{R N}^{\mathrm{T}}}_{\mathbf{I}_{R}} \mathbf{r}_{v}(t)+\underbrace{\mathbf{R} \mathbf{W}_{i n}}_{\mathbf{0}_{R \times p}} \mathbf{u}_{i n}(t)+\underbrace{\mathbf{R} \mathbf{n}_{0}}_{\mathbf{0}_{R}} \delta(t)-\omega(t) \mathbf{x}_{r}(t) & \mathbf{x}_{r}(0)=\mathbf{0}_{R} \\
\dot{\mathbf{x}}_{i n}(t)=\underbrace{\mathbf{F N}^{\mathrm{T}}}_{\mathbf{0}_{p \times R}} \mathbf{r}_{v}(t)+\underbrace{\mathbf{F} \mathbf{W}_{i n}}_{\mathbf{I}_{p}} \mathbf{u}_{i n}(t)+\underbrace{\mathbf{F} \mathbf{n}_{0}}_{\mathbf{0}_{p}} \delta(t)-\omega(t) \mathbf{x}_{i n}(t) & \mathbf{x}_{i n}(0)=\mathbf{0}_{p} \\
\dot{\mathbf{x}}_{i c}(t)=\underbrace{\mathbf{i}^{\mathrm{T}} \mathbf{N}^{\mathrm{T}}}_{\mathbf{0}_{R}^{\mathrm{T}}} \mathbf{r}_{v}(t)+\underbrace{\mathbf{i}^{\mathrm{T}} \mathbf{W}_{i n}}_{\mathbf{0}_{p}^{\mathrm{T}}} \mathbf{u}_{i n}(t)+\underbrace{\mathbf{i}^{\mathrm{T}} \mathbf{n}_{0}}_{1} \delta(t)-\omega(t) x_{i c}(t) & x_{i c}(0)=0 \\
\dot{\mathbf{x}}_{i v}(t)=\underbrace{\mathbf{Q N}^{\mathrm{T}}}_{\mathbf{0}_{q \times R}} \mathbf{r}_{v}(t)+\underbrace{\mathbf{Q} \mathbf{W}_{i n}}_{\mathbf{0}_{q \times p}} \mathbf{u}_{i n}(t)+\underbrace{\mathbf{Q} \mathbf{n}_{0}}_{\mathbf{0}_{q}} \delta(t)-\omega(t) \mathbf{x}_{i v}(t) & \mathbf{x}_{i v}(0)=\mathbf{0}_{q},
\end{array}
$$

where $\mathbf{R}, \mathbf{F}$ and $\mathbf{Q}$ are matrices of dimension $R \times S, p \times S$, and $q \times S$, respectively, and $\mathbf{i}$ is a $S$-dimensional vector, with $q=S-R-p-1$ being the number of invariant quantities.

Upon choosing $\mathcal{T}$ as

$$
\mathcal{T}=\left[\begin{array}{llll}
\mathbf{N}^{\mathrm{T}} & \mathbf{W}_{\text {in }} & \mathbf{n}_{0} & \mathbf{P}
\end{array}\right]^{-1},
$$

\footnotetext{
${ }^{2}$ For example, $\dot{\mathbf{n}}(t)=\mathbf{0}_{S}, \mathbf{n}(0)=\mathbf{n}_{0}$ can be written equivalently as $\dot{\mathbf{n}}(t)=\mathbf{n}_{0} \delta(t)$, $\mathbf{n}(0)=\mathbf{0}_{S}$.

3 Another way to have zero initial conditions is to work in terms of deviation variables as shown in Appendix A.
} 


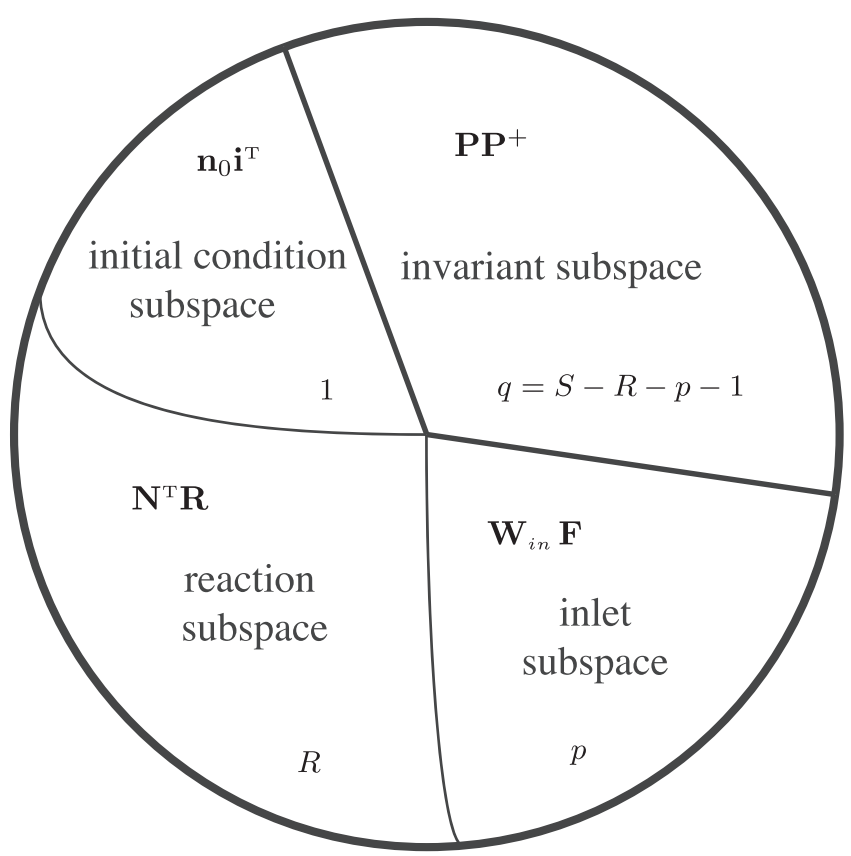

Fig. 1. Decomposition of the $S$-dimensional subspace of numbers of moles into an $R$ dimensional reaction subspace, a $p$-dimensional inlet subspace, a one-dimensional subspace describing the contribution of the initial conditions and a $q$-dimensional invariant subspace.

where the $S \times q$ matrix $\mathbf{P}$ describes the $q$-dimensional null space of the matrix [ $\left.\begin{array}{lll}\mathbf{N}^{\mathrm{T}} & \mathbf{W}_{\text {in }} & \mathbf{n}_{0}\end{array}\right]^{\mathrm{T}},{ }^{4}$ the transformation gives the conditions shown under the braces in Eq. (10), namely:

$\left[\begin{array}{l}\mathbf{R} \\ \mathbf{F} \\ \mathbf{i}^{\mathrm{T}} \\ \mathbf{Q}\end{array}\right]\left[\begin{array}{llll}\mathbf{N}^{\mathrm{T}} & \mathbf{W}_{i n} & \mathbf{n}_{0} & \mathbf{P}\end{array}\right]=\left[\begin{array}{cccc}\mathbf{I}_{R} & \mathbf{0} & \mathbf{0} & \mathbf{0} \\ \mathbf{0} & \mathbf{I}_{p} & \mathbf{0} & \mathbf{0} \\ \mathbf{0} & \mathbf{0} & 1 & \mathbf{0} \\ \mathbf{0} & \mathbf{0} & \mathbf{0} & \mathbf{I}_{q}\end{array}\right]$.

It follows from $\mathbf{P}$ being orthogonal to $\left[\mathbf{N}^{\mathrm{T}} \mathbf{W}_{i n} \mathbf{n}_{0}\right]$ and $\mathbf{Q P}=\mathbf{I}_{q}$ that $\mathbf{Q}=\mathbf{P}^{+}$. Furthermore, $\mathbf{N}^{\mathrm{T}} \mathbf{R}+\mathbf{W}_{i n} \mathbf{F}+\mathbf{n}_{0} \mathbf{i}^{\mathrm{T}}+\mathbf{P} \mathbf{P}^{+}=\mathbf{I}_{S}$, where $\mathbf{N}^{\mathrm{T}} \mathbf{R}$ represents the $R$-dimensional reaction subspace, $\mathbf{W}_{i n} \mathbf{F}$ the $p$ dimensional inlet subspace, $\mathbf{n}_{0} \mathbf{i}^{\mathrm{T}}$ the one-dimensional subspace describing the contribution of the initial conditions, and $\mathbf{P} \mathbf{P}^{+}$the $q$-dimensional invariant subspace (Fig. 1). All subspaces add up to the $S$-dimensional species space $\mathbb{R}^{S}$. Note that the invariant subspace is orthogonal to the other subspaces by construction, while the other subspaces are typically not orthogonal to each other.

\subsubsection{Vessel extents and invariants}

If $\operatorname{rank}\left(\left[\mathbf{N}^{\mathrm{T}} \mathbf{W}_{\text {in }} \mathbf{n}_{0}\right]\right)=R+p+1$, the linear transformation (11) brings the dynamic model (8) to: ${ }^{5}$

$$
\begin{aligned}
& \dot{x}_{r, i}(t)=r_{v, i}(t)-\omega(t) x_{r, i}(t) \quad x_{r, i}(0)=0 \quad i=1, \ldots, R \\
& \dot{x}_{i n, j}(t)=u_{i n, j}(t)-\omega(t) x_{i n, j}(t) \quad x_{i n, j}(0)=0 \quad j=1, \ldots, p \\
& \dot{x}_{i c}(t)=-\omega(t) x_{i c}(t) \quad x_{i c}(0)=1 \\
& \mathbf{x}_{i v}(t)=\mathbf{0}_{q},
\end{aligned}
$$

where $x_{r, i}(t)$ is the extent of the $i$ th reaction at time $t$ expressed in $\mathrm{kmol}, x_{i n, j}(t)$ the extent of the $j$ th inlet flow at time $t$ expressed in

${ }^{4}\left[\begin{array}{lll}\mathbf{N}^{\mathrm{T}} & \mathbf{W}_{\text {in }} & \mathbf{n}_{0}\end{array}\right]^{\mathrm{T}} \mathbf{P}=\mathbf{0}_{(S-q) \times q}$ or, equivalently, $\mathbf{P}^{\mathrm{T}}\left[\mathbf{N}^{\mathrm{T}} \mathbf{W}_{i n} \mathbf{n}_{0}\right]=\mathbf{0}_{q \times(S-q)}$. In this case, the matrix $\mathbf{P}$ spans the space that is orthogonal to the reactions, the inlets and the initial conditions.

5 The differential equation $\dot{x}_{i c}(t)=\delta(t)-\omega(t) x_{i c}(t), x_{i c}(0)=0$, can be written as Eq. (13c), which is easier to simulate. $\mathrm{kg}, x_{i c}(t)$ the dimensionless extent of initial conditions, which jumps from 0 to 1 and indicates the fraction of the initial conditions that is still in the reactor at time $t$, and $\mathbf{x}_{i v}(t)$ the vector of invariants at time $t$. Note that each extent is affected by its corresponding rate process $\left(r_{v, i}(t), u_{i n, j}(t), \delta(t)\right)$ and, in the presence of an outlet $(\omega(t)>0)$, also by the inlet and outlet flows. Since each extent is discounted by the amount that has left the reactor and thus represents the amount of material associated with the corresponding rate that is still in the vessel, these extents are called "vessel extents". The numbers of moles $\mathbf{n}(t)$ can be reconstructed from the various extents by premultiplying (9) by $\mathcal{T}^{-1}=\left[\begin{array}{llll}\mathbf{N}^{\mathrm{T}} & \mathbf{W}_{i n} & \mathbf{n}_{0} & \mathbf{P}\end{array}\right]$ and considering the fact that $\mathbf{x}_{i v}(t)=\mathbf{0}_{q}$ :

$\mathbf{n}(t)=\mathbf{N}^{\mathrm{T}} \mathbf{x}_{r}(t)+\mathbf{W}_{i n} \mathbf{x}_{i n}(t)+\mathbf{n}_{0} x_{i c}(t)$.

Note that the mass $m(t)$ can also be reconstructed from $\mathbf{x}_{i n}(t)$ and $x_{i c}(t)$ as follows:

$m(t)=\mathbf{1}_{p}^{\mathrm{T}} \mathbf{x}_{i n}(t)+m_{0} x_{i c}(t)$.

\section{Remarks}

- The invariants $\mathbf{x}_{i v}(t)$ are identically equal to zero and can be discarded from the model. The invariant relationships $\mathbf{P}^{+} \mathbf{n}(t)=\mathbf{0}_{q}$, which represent $q$ constraints prevailing among the variables $\mathbf{n}(t)$, can be rather useful in practice to reduce the number of degrees of freedom in a given problem.

- The extents $\mathbf{x}_{i n}(t)$ and $x_{i c}(t)$ can be computed from $\mathbf{u}_{i n}(t)$ and $u_{\text {out }}(t)$ in Eqs. (13b) and (13c) and the continuity Eq. (2):

$$
\begin{aligned}
\dot{\mathbf{x}}_{i n}(t) & =\mathbf{u}_{\text {in }}(t)-\omega(t) \mathbf{x}_{\text {in }}(t) & \mathbf{x}_{i n}(0) & =\mathbf{0}_{p} \\
\dot{x}_{i c}(t) & =-\omega(t) x_{i c}(t) & x_{i c}(0) & =1 \\
\dot{m}(t) & =\mathbf{1}_{p}^{\mathrm{T}} \mathbf{u}_{\text {in }}(t)-u_{\text {out }}(t), & & m(0)=m_{0} .
\end{aligned}
$$

- The extent of reaction $x_{r, i}(t)$ depends only upon the corresponding reaction rate $r_{v, i}(t)$ and the inlet and outlet flows, but not on the other rate processes. It follows that $r_{v, i}(t)$ can be computed directly from $x_{r, i}(t)$, its time derivative and $\omega(t)$, that is, without having to know the other extents. However, this apparent decoupling is somewhat misleading: indeed, since $r_{v, i}(t)$ is an endogenous signal (and not an exogenous input), it also depends on what happens in the reactor, that is, it feels the effect of most other processes. Hence, the prevailing decoupling is limited to the relationships between rate and extent signals, as given in (13a)-(13c).

\subsection{Special cases}

We consider next the transformed systems for three special cases, namely, batch reactors, semi-batch reactors and CSTRs.

\subsubsection{Batch reactors}

In a batch reactor with $p=0$ and no outlet, the linear transformation (11) reduces to:

$\mathcal{T}=\left[\begin{array}{lll}\mathbf{N}^{\mathrm{T}} & \mathbf{n}_{0} & \mathbf{P}\end{array}\right]^{-1}$,

with $q=S-R-1$, and the transformed system reads:

$\dot{x}_{r, i}(t)=r_{v, i}(t) \quad x_{r, i}(0)=0 \quad i=1, \ldots, R$

$x_{i c}(t)=1$

$\mathbf{x}_{i v}(t)=\mathbf{0}_{q}$.

Note that, since there is no outlet, $x_{i c}=1$ is also invariant. Hence, there are $R$ reaction variants and $S-R$ invariants. The numbers of moles $\mathbf{n}(t)$ can be expressed as:

$\mathbf{n}(t)=\mathbf{N}^{\mathrm{T}} \mathbf{x}_{r}(t)+\mathbf{n}_{0}$. 


\subsubsection{Semi-batch reactors}

A semi-batch reactor has $p$ inlets but no outlet. With the linear transformation (11), the transformed system becomes:

$$
\begin{aligned}
& \dot{x}_{r, i}(t)=r_{v, i}(t) \quad x_{r, i}(0)=0 \quad i=1, \ldots, R \\
& \dot{x}_{i n, j}(t)=u_{i n, j}(t) \quad x_{i n, j}(0)=0 \quad j=1, \ldots, p \\
& x_{i c}(t)=1 \\
& \mathbf{x}_{i v}(t)=\mathbf{0}_{q},
\end{aligned}
$$

with $q=S-R-p-1$. There are $R$ reaction variants, $p$ inlet variants and $S-R-p$ invariants. The numbers of moles $\mathbf{n}(t)$ can be expressed as:

$\mathbf{n}(t)=\mathbf{N}^{\mathrm{T}} \mathbf{x}_{r}(t)+\mathbf{W}_{i n} \mathbf{x}_{i n}(t)+\mathbf{n}_{0}$.

\subsubsection{Continuous stirred-tank reactors (CSTR)}

In a CSTR, $u_{\text {out }}(t)$ is computed from Eq. (2) and $m(t)=V_{0} \rho(t)$, with $V_{0}$ the constant volume, as follows:

$u_{\text {out }}(t)=\mathbf{1}_{p}^{\mathrm{T}} \mathbf{u}_{\text {in }}(t)-V_{0} \dot{\rho}(t)$.

- If the density varies, the transformed system (13a)-(13d) cannot be simplified and thus holds with $q=S-R-p-1$.

- If the density is constant, $u_{\text {out }}(t)=\mathbf{1}_{p}^{\mathrm{T}} \mathbf{u}_{\text {in }}(t)$ and thus $\omega(t)=$ $\left(\mathbf{1}_{p}^{\mathrm{T}} \mathbf{u}_{i n}(t)\right) / m_{0}$. In this case, $x_{i c}(t)$ can be computed algebraically from the states $\mathbf{x}_{i n}(t)$ as $x_{i c}(t)=1-\left(\mathbf{1}_{p}^{\mathrm{T}} \mathbf{x}_{i n}(t)\right) / m_{0}$, with $m_{0}=V_{0} \rho$, which can be shown by differentiating the last expression and writing $\dot{\mathbf{x}}_{i n}(t)$ and $\dot{x}_{i c}(t)$ using Eqs. (13b) and (13c). The transformed system becomes:

$$
\begin{array}{rlrl}
\dot{x}_{r, i}(t) & =r_{v, i}(t)-\omega(t) x_{r, i}(t) & x_{r, i}(0)=0 & i=1, \ldots, R \\
\dot{x}_{i n, j}(t) & =u_{i n, j}(t)-\omega(t) x_{i n, j}(t) & x_{i n, j}(0)=0 & j=1, \ldots, p \\
x_{i c}(t) & =1-\frac{\mathbf{1}_{p}^{\mathrm{T}} \mathbf{x}_{i n}(t)}{m_{0}} & & \\
\mathbf{x}_{i v}(t) & =\mathbf{0}_{q} . & &
\end{array}
$$

Since $x_{i c}(t)$ is linearly dependent on $\mathbf{x}_{i n}(t)$, the system is of order $R+p, q=S-R-p-1$, and there are $q+1=S-R-p$ invariants. The numbers of moles $\mathbf{n}(t)$ can be expressed as:

$\mathbf{n}(t)=\mathbf{N}^{\mathrm{T}} \mathbf{x}_{r}(t)+\left(\mathbf{W}_{i n}-\frac{\mathbf{n}_{0} \mathbf{1}_{p}^{\mathrm{T}}}{m_{0}}\right) \mathbf{x}_{i n}(t)+\mathbf{n}_{0}$.

\section{Homogeneous reaction systems with heat balance}

Let us consider an open non-isothermal homogeneous reactor that involves heat exchange via a heating/cooling jacket.

\subsection{Mole and heat balance equations}

The model includes the mole balance Eq. (1a) and a heat balance around the reactor (Amrhein, 1998):

$$
\begin{array}{ll}
\dot{\mathbf{n}}(t)=\mathbf{N}^{\mathrm{T}} \mathbf{r}_{v}(t)+\mathbf{W}_{i n} \mathbf{u}_{i n}(t)-\omega(t) \mathbf{n}(t) & \mathbf{n}(0)=\mathbf{n}_{0} \\
\dot{Q}(t)=(-\Delta \mathbf{H})^{\mathrm{T}} \mathbf{r}_{v}(t)+q_{e x}(t)+\check{\mathbf{T}}_{i n}^{\mathrm{T}} \mathbf{u}_{i n}(t)-\omega(t) Q(t) & Q(0)=Q_{0},
\end{array}
$$

where $Q=m c_{p} T$ is the heat of the reaction mixture, with $T$ the reactor temperature and $c_{p}$ the specific heat capacity, $q_{e x}$ is the heat flow from the jacket to the reaction mixture, $\check{\mathbf{T}}_{i n}$ the $p$-dimensional vector of specific heat of the inlet streams with $\check{T}_{i n, j}=c_{p, i n, j} T_{i n, j}$ and $T_{i n, j}$ the temperature of the $j$ th inlet, and $\Delta \mathbf{H}$ the $R$-dimensional vector of reaction enthalpies. Obviously, the heat flow term $q_{\text {ex }}(t)$ depends on the reactor temperature, for example $q_{e x}(t)=U A\left(T_{j}(t)-T(t)\right)$ with the heat transfer coefficient $U A$ and the jacket temperature $T_{j}(t)$, but this dependency is not needed at the level of Eq. (25). This model assumes that most physical properties are not temperature dependent, which is well justified for small temperature variations. Furthermore, for simplicity, let us assume that the inlet specific heat vector $\check{\mathbf{T}}_{i n}$ is constant.

\subsection{Vessel extents and invariants}

For the sake of deriving the transformation, we will express the initial conditions as a unit impulse at time 0 . The model can be written in compact form using the $(S+1)$-dimensional state vector $\mathbf{z}(t)=\left[\begin{array}{l}\mathbf{n}(t) \\ Q(t)\end{array}\right]$

$\dot{\mathbf{z}}(t)=\mathcal{A} \mathbf{r}_{v}(t)+\mathbf{b} q_{e x}(t)+\mathcal{C} \mathbf{u}_{i n}(t)+\mathbf{z}_{0} \delta(t)-\omega(t) \mathbf{z}(t) \quad \mathbf{z}(0)=\mathbf{0}_{S+1}$,

where $\mathcal{A}=\left[\begin{array}{c}\mathbf{N}^{\mathrm{T}} \\ (-\Delta \mathbf{H})^{\mathrm{T}}\end{array}\right], \mathbf{b}=\left[\begin{array}{l}\mathbf{0}_{S} \\ 1\end{array}\right], \mathcal{C}=\left[\begin{array}{l}\mathbf{W}_{\text {in }} \\ \check{\mathbf{T}}_{\text {in }}^{\mathrm{T}}\end{array}\right]$ and $\mathbf{z}_{0}=\left[\begin{array}{c}\mathbf{n}_{0} \\ \mathrm{Q}_{0}\end{array}\right]$.

The right-hand side of Eq. (26) has five contributions that indicate the effects of the reactions, the heat exchange, the inlets, the initial conditions, and the outlet, respectively.

\subsubsection{Decoupling transformation}

The linear transformation $\mathcal{T}=\left[\begin{array}{lllll}\mathcal{A} & \mathbf{b} & \mathcal{C} & \mathbf{z}_{0} & \mathbf{P}\end{array}\right]^{-1}$ transforms $\mathbf{z}(t)$ into five parts, namely, $\mathbf{x}_{r}(t), x_{e x}(t), \mathbf{x}_{i n}(t)$ and $x_{i c}(t)$ that are associated with the reactions, the heat exchange, the inlets and the initial conditions, and $\mathbf{x}_{i v}(t)$ that is orthogonal to the other extents and invariant as will be seen below:

$$
\left[\begin{array}{c}
\mathbf{x}_{r}(t) \\
x_{e x}(t) \\
\mathbf{x}_{i n}(t) \\
x_{i c}(t) \\
\mathbf{x}_{i v}(t)
\end{array}\right]=\mathcal{T} \mathbf{z}(t):=\left[\begin{array}{c}
\mathbf{R} \\
\mathbf{h}^{\mathrm{T}} \\
\mathbf{F} \\
\mathbf{i}^{\mathrm{T}} \\
\mathbf{P}^{+}
\end{array}\right] \mathbf{z}(t) .
$$

The $S \times q$ matrix $\mathbf{P}$ describes the $q$-dimensional null space of the matrix $\left[\begin{array}{llll}\mathcal{A} & \mathbf{b} & \mathcal{C} & \mathbf{z}_{0}\end{array}\right]^{\mathrm{T}}$, with $q=S-R-p-1$.

\subsubsection{Vessel extents and invariants}

If $\operatorname{rank}\left(\left[\begin{array}{llll}\mathcal{A} & \mathbf{b} & \mathcal{C} & \mathbf{z}_{0}\end{array}\right]\right)=R+p+2$, the linear transformation (27) brings the dynamic model (26) to:

$$
\begin{array}{ll}
\dot{\mathrm{x}}_{r}(t)=\mathbf{r}_{v}(t)-\omega(t) \mathbf{x}_{r}(t) & \mathbf{x}_{r}(0)=\mathbf{0}_{R} \\
\dot{x}_{e x}(t)=q_{e x}(t)-\omega(t) x_{e x}(t) & x_{e x}(0)=0 \\
\dot{\mathrm{x}}_{i n}(t)=\mathbf{u}_{i n}(t)-\omega(t) \mathbf{x}_{i n}(t) & \mathbf{x}_{i n}(0)=\mathbf{0}_{p} \\
\dot{x}_{i c}(t)=-\omega(t) x_{i c}(t) & x_{i c}(0)=1 \\
\mathbf{x}_{i v}(t)=\mathbf{0}_{q}, &
\end{array}
$$

where $x_{e x}$ is the extent of heat exchange expressed in kJ. Compared to the transformed model (13a)-(13d), the model (28) has the one-dimensional state $x_{e x}(t)$ to describe the evolution of the heat exchange. Note that the extents $\mathbf{x}_{r}, \mathbf{x}_{i n}$ and $x_{i c}$ in Eq. (28) are those in Eqs. (13a)-(13c), which confirms the fact that the transformed model (13a)-(13c) can be used to describe the reactions and flows in a non-isothermal reactor also in the absence of a heat balance. 
The numbers of moles $\mathbf{n}(t)$ and the heat $Q(t)$ can be reconstructed from the transformed variables as follows:

$\mathbf{z}(t)=\mathcal{A} \mathbf{x}_{r}(t)+\mathbf{b} x_{e x}(t)+\mathcal{C} \mathbf{x}_{i n}(t)+\mathbf{z}_{0} x_{i c}(t)$.

A possible use of this decoupling regards the estimation of $q_{e x}(t)$ or the identification of the heat-transfer coefficient $U A$ in the expression $q_{e x}(t)=U A\left(T_{j}(t)-T(t)\right)$, independently of any kinetic information, from discrete measurements of $\mathbf{z}(t)$ and computation of $x_{e x}(t)$ via the transformation (27).

\section{Fluid-fluid reaction systems}

This section extends the results obtained for homogeneous reaction systems to heterogeneous fluid-fluid reaction systems. In addition to the extents developed above, there will be extents of mass transfer that describe the material transport between the two phases.

We consider here a reaction system consisting of two phases, namely, the $G$ and $L$ phases. ${ }^{6}$ The two phases are modeled separately, with the mass-transfer rates $\zeta$ connecting the two phases. The $L$ phase contains $S_{l}$ species, $p_{l}$ inlets and one outlet, while the $G$ phase contains $S_{g}$ species, $p_{g}$ inlets and one outlet. There are $p_{m}$ mass transfers taking place between the two phases. The reactions can occur in both phases, with $R_{l}$ reactions in phase $L$ and $R_{g}$ reactions in phase $G$.

\subsection{Mole balance equations} read:

The differential mole balance equations for phase $F, F \in\{G, L\}$,

$$
\begin{array}{r}
\dot{\mathbf{n}}_{f}(t)=\mathbf{N}_{f}^{\mathrm{T}} \mathbf{r}_{v, f}(t) \pm \mathbf{W}_{m, f} \zeta(t)+\mathbf{W}_{i n, f} \mathbf{u}_{i n, f}(t)-\omega_{f}(t) \mathbf{n}_{f}(t) \\
\mathbf{n}_{f}(0)=\mathbf{n}_{f 0},
\end{array}
$$

with a positive sign (+) for phase $L$ and a negative sign $(-)$ for phase $G$, and where the subscript $(.)_{f}$ is used to denote phase $F$, with $f \in\{g, l\}$. The $p_{m}$ mass transfers are treated as pseudo inlets with the mass-transfer rates $\boldsymbol{\zeta}$, and $\mathbf{W}_{m, f}=\mathbf{M}_{w, f}^{-1} \mathbf{E}_{m, f}$ is the $S_{f} \times p_{m}$ mass-transfer matrix, $\mathbf{E}_{m, f}=\left[\begin{array}{lll}\mathbf{e}_{m, f}^{1} & \ldots & \mathbf{e}_{m, f}^{p_{m}}\end{array}\right]$ with $\mathbf{e}_{m, f}^{j}$ being the $S_{f}$-dimensional vector with the element corresponding to the $j$ th transferring species equal to unity and the other elements equal to zero.

\subsection{Vessel extents and invariants}

Again, we will express the initial conditions as a unit impulse at time 0 , which gives the following balance equations:

$$
\begin{array}{r}
\dot{\mathbf{n}}_{f}(t)=\mathbf{N}_{f}^{\mathrm{T}} \mathbf{r}_{v, f}(t) \pm \mathbf{W}_{m, f} \zeta(t)+\mathbf{W}_{i n, f} \mathbf{u}_{i n, f}(t)+\mathbf{n}_{f 0} \delta(t)-\omega_{f}(t) \mathbf{n}_{f}(t) \\
\mathbf{n}_{f}(0)=\mathbf{0}_{S_{f}} .
\end{array}
$$

The right-hand side of Eq. (31) has five contributions that indicate the effects of the reactions, the mass transfers, the inlets, the initial conditions, and the outlet, respectively.

\subsubsection{Decoupling transformation}

For phase $F$, the linear transformation $\mathcal{I}_{f}:=$ $\left[\begin{array}{lllll}\mathbf{N}_{f}^{\mathrm{T}} & \pm \mathbf{W}_{m, f} & \mathbf{W}_{i n, f} & \mathbf{n}_{f 0} & \mathbf{P}_{f}\end{array}\right]^{-1}$ transforms $\mathbf{n}_{f}(t)$ into five parts, namely, $\mathbf{x}_{r, f}(t), \mathbf{x}_{m, f}(t), \mathbf{x}_{i n f}(t)$ and $x_{i c, f}(t)$ that are associated with the reactions, the mass transfers, the inlets and the initial conditions,

${ }^{6}$ Although $G$ and $L$ are often the gas and liquid phases, they can also refer to two distinct liquid phases. and $\mathbf{x}_{i v f}(t)$ that is orthogonal to the other extents and invariant as will be seen below:

$$
\left[\begin{array}{c}
\mathbf{x}_{r, f}(t) \\
\mathbf{x}_{m, f}(t) \\
\mathbf{x}_{i n, f}(t) \\
x_{i c, f}(t) \\
\mathbf{x}_{i v, f}(t)
\end{array}\right]=\left[\begin{array}{lllll}
\mathbf{N}_{f}^{\mathrm{T}} & \pm \mathbf{W}_{m, f} & \mathbf{W}_{i n, f} & \mathbf{n}_{f 0} & \mathbf{P}_{f}
\end{array}\right]^{-1} \mathbf{n}_{f}(t),
$$

where the matrix $\mathbf{P}_{f}$ of dimension $S_{f} \times q_{f}$ describes the $q_{f^{-}}$ dimensional null space of the matrix $\left[\begin{array}{llll}\mathbf{N}_{f}^{\mathrm{T}} & \pm \mathbf{W}_{m, f} & \mathbf{W}_{i n, f} & \mathbf{n}_{f 0}\end{array}\right]^{\mathrm{T}}$, with $q_{f}=S_{f}-R_{f}-p_{m}-p_{f}-1$.

\subsubsection{Vessel extents and invariants}

If $\operatorname{rank}\left(\left[\mathbf{N}_{f}^{\mathrm{T}} \pm \mathbf{W}_{m, f} \mathbf{W}_{i n, f} \quad \mathbf{n}_{f 0}\right]\right)=R_{f}+p_{m}+p_{f}+1$, the linear transformation (32) brings (31) to:

$$
\begin{array}{ll}
\dot{\mathbf{x}}_{r, f}(t)=\mathbf{r}_{v, f}(t)-\omega_{f}(t) \mathbf{x}_{r, f}(t) & \mathbf{x}_{r, f}(0)=\mathbf{0}_{R_{f}} \\
\dot{\mathbf{x}}_{m, f}(t)=\zeta(t)-\omega_{f}(t) \mathbf{x}_{m, f}(t) & \mathbf{x}_{m, f}(0)=\mathbf{0}_{p_{m}} \\
\dot{\mathbf{x}}_{i n, f}(t)=\mathbf{u}_{i n, f}(t)-\omega_{f}(t) \mathbf{x}_{i n, f}(t) & \mathbf{x}_{i n, f}(0)=\mathbf{0}_{p_{f}} \\
\dot{x}_{i c, f}(t)=-\omega_{f}(t) x_{i c, f}(t) & x_{i c, f}(0)=1 \\
\mathbf{x}_{i v, f}(t)=\mathbf{0}_{q_{f}} . &
\end{array}
$$

Compared to the transformed model (13a)-(13d), the model (33) has the $p_{m}$-dimensional states $\mathbf{x}_{m, f}(t)$ to describe the evolution of the mass transfers. The reconstruction of the numbers of moles $\mathbf{n}_{f}(t)$ reads:

$$
\mathbf{n}_{f}(t)=\mathbf{N}_{f}^{\mathrm{T}} \mathbf{x}_{r, f}(t) \pm \mathbf{W}_{m, f} \mathbf{x}_{m, f}(t)+\mathbf{W}_{i n, f} \mathbf{x}_{i n, f}(t)+\mathbf{n}_{f 0} x_{i c, f}(t) .
$$

\section{Distributed reaction systems}

For lumped homogeneous reaction systems, the transformation (9) is able to isolate the contributions of the reactions, inlets and initial conditions that are contained in the state vector $\mathbf{n}(t)$. In addition, it is possible to express the invariant states as $\mathbf{P}^{+} \mathbf{n}(t)=\mathbf{0}_{q}$, which is useful in many applications (Waller and Mäkilä, 1981; Fjeld et al., 1974; Gadewar et al., 2002). We develop next a similar transformation applicable to distributed reaction systems. As illustrative example, we will consider a one-dimensional single-phase tubular reactor model (Friedly, 1972).

\subsection{Material balance equations}

Consider the reaction-convection-diffusion system

$\frac{\partial \mathbf{c}(z, t)}{\partial t}=\mathbf{N}^{\mathrm{T}} \mathbf{r}(z, t)+\mathbf{E}_{d} \boldsymbol{\phi}(z, t)-\frac{\partial(v(z, t) \mathbf{c}(z, t))}{\partial z}$,

with the initial conditions $\mathbf{c}(z, 0)=\mathbf{c}_{0}(z)$ and the boundary conditions $\mathbf{c}(0, t)=\mathbf{c}_{i n}(t)$ and $\lim _{z \rightarrow \infty}(\partial \mathbf{c}(z, t) / \partial z)=\mathbf{0}_{S} \cdot{ }^{7}$ Here, $\mathbf{c}(z, t)$ represents the $S$-dimensional vector of concentrations at the spatial coordinate $z$ and time $t$ in $\mathrm{kmol} / \mathrm{m}^{3}, v(z, t)$ is the velocity of the convective flow in $\mathrm{m} / \mathrm{s}$, and $\phi(z, t)$ is the $p_{d}$-dimensional vector of diffusion rates in $\mathrm{kmol} /\left(\mathrm{m}^{3} \mathrm{~s}\right)$. It is assumed that only $p_{d} \leq S-R$ species are affected by diffusion as indicated by the $S \times p_{d}$ matrix

\footnotetext{
7 These boundary conditions correspond to what has been called "approximation by a reactor of infinite length" (Fan and Ahn, 1962), since the boundary conditions are given at $z=0$ and $z \rightarrow \infty$. Note that the separation of the various effects can also be achieved when different boundary conditions are assumed, as shown in Appendix B for the case of the Danckwerts boundary conditions (Danckwerts, 1953).
} 
$\mathbf{E}_{d}=\left[\begin{array}{lll}\mathbf{e}_{d}^{1} & \cdots & \mathbf{e}_{d}^{p_{d}}\end{array}\right]$, with $\mathbf{e}_{d}^{j}$ being the $S$-dimensional vector with the element corresponding to the $j$ th diffusive species equal to unity and the other elements equal to zero. This model includes $S$ species, $R$ independent reactions, $p_{d}$ diffusive terms and one convective term. The reaction and diffusion rates are modeled as the unknown signals $\mathbf{r}(z, t)$ and $\phi(z, t)$, which hides the fact that the rates depend on $z$ and $t$ over the concentrations $\mathbf{c}(z, t)$ and the temperature $T(z$, $t)$.

The right-hand side of Eq. (35) has three contributions that are associated with reactions, diffusion and convection. Since the corresponding terms $\mathbf{N}^{\mathrm{T}} \mathbf{r}(z, t), \mathbf{E}_{d} \boldsymbol{\phi}(z, t)$ and $\partial(v(z, t) \mathbf{c}(z, t)) / \partial z$ appear linearly, the principle of superposition is satisfied and each contribution can be computed separately. We will first remove the contribution of the reactions and diffusion and compute the effect that convection has on the initial and boundary conditions by solving the differential equation

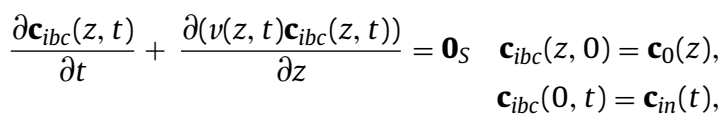

where $\mathbf{c}_{i b c}(z, t)$ indicates the concentrations at position $z$ and time $t$ that are due uniquely to the non-zero initial and convective boundary conditions.

We will then remove the effect of the initial and boundary conditions by writing the concentrations as deviations from $\mathbf{c}_{i b c}(z, t)$,

$\delta \mathbf{c}(z, t):=\mathbf{c}(z, t)-\mathbf{c}_{i b c}(z, t)$,

with which Eq. (35) becomes:

$\frac{\partial \delta \mathbf{c}(z, t)}{\partial t}=\mathbf{N}^{\mathrm{T}} \mathbf{r}(z, t)+\mathbf{E}_{d} \boldsymbol{\phi}(z, t)-\frac{\partial(v(z, t) \delta \mathbf{c}(z, t))}{\partial z}$,

with the initial conditions $\delta \mathbf{c}(z, 0)=\mathbf{0}_{S}$ and the boundary conditions $\delta \mathbf{c}(0, t)=\mathbf{0}_{S}$ and $\lim _{z \rightarrow \infty}(\partial \delta \mathbf{c}(z, t) / \partial z)=\mathbf{0}_{S} .{ }^{8}$ The right-hand side of Eq. (38) has three contributions, with the first two associated with the $R$ reactions and the $p_{d}$ diffusion terms, respectively.

\subsection{Extents and invariants}

This section proposes a transformation that generates $R$ extents of reaction, $p_{d}$ extents of diffusion and, as will be seen, $q=S-R-p_{d}$ invariants.

\subsubsection{Decoupling transformation}

The linear transformation $\mathcal{T}=\left[\begin{array}{lll}\mathbf{N}^{\mathrm{T}} & \mathbf{E}_{d} & \mathbf{P}\end{array}\right]^{-1}$ transforms $\delta \mathbf{c}(z, t)$ into three parts, namely, $\mathbf{x}_{r}(z, t)$ and $\mathbf{x}_{d}(z, t)$ that are associated with the reactions and the diffusion, and the part $\mathbf{x}_{i v}(z, t)$ that is invariant as will be seen below:

$$
\left[\begin{array}{l}
\mathbf{x}_{r}(z, t) \\
\mathbf{x}_{d}(z, t) \\
\mathbf{x}_{i v}(z, t)
\end{array}\right]=\mathcal{T} \delta \mathbf{c}(z, t):=\left[\begin{array}{l}
\mathbf{R} \\
\mathbf{D} \\
\mathbf{P}^{+}
\end{array}\right] \delta \mathbf{c}(z, t)
$$

where the $S \times q$ matrix $\mathbf{P}$ describes the $q$-dimensional null space of the matrix $\left[\begin{array}{ll}\mathbf{N}^{\mathrm{T}} & \mathbf{E}_{d}\end{array}\right]^{\mathrm{T}}$.

\footnotetext{
8 The last boundary condition follows from the assumption $\lim \left(\partial \mathbf{c}_{i b c}(z, t) / \partial z\right)=$
} $\mathbf{0}_{S}$

\subsubsection{Extents and invariants}

If $\operatorname{rank}\left(\left[\mathbf{N}^{\mathrm{T}} \mathbf{E}_{d}\right]\right)=R+p_{d}$, the linear transformation (39) brings the dynamic model (38) to:

$$
\begin{aligned}
\frac{\partial \mathbf{x}_{r}(z, t)}{\partial t}+\frac{\partial\left(v(z, t) \mathbf{x}_{r}(z, t)\right)}{\partial z}=\mathbf{r}(z, t) & \mathbf{x}_{r}(z, 0)=\mathbf{0}_{R}, \\
\mathbf{x}_{r}(0, t) & =\mathbf{0}_{R} \\
\frac{\partial \mathbf{x}_{d}(z, t)}{\partial t}+\frac{\partial\left(v(z, t) \mathbf{x}_{d}(z, t)\right)}{\partial z}=\boldsymbol{\phi}(z, t) \quad \mathbf{x}_{d}(z, 0) & =\mathbf{0}_{p_{d}}, \\
\mathbf{x}_{d}(0, t)=\mathbf{0}_{p_{d}}, \quad \lim _{z \rightarrow \infty} \frac{\partial \mathbf{x}_{d}(z, t)}{\partial z} & =\mathbf{0}_{p_{d}}
\end{aligned}
$$

$\mathbf{x}_{i v}(z, t)=\mathbf{0}_{q}$,

where $\mathbf{x}_{r}(z, t)$ indicates the change in concentrations at position $z$ and time $t$ due to the reactions; $\mathbf{x}_{d}(z, t)$ indicates the change in concentrations at position $z$ and time $t$ due to diffusion; $\mathbf{x}_{i v}(z, t)$ represents variables that are orthogonal to these extents and therefore invariant. The concentrations $\delta \mathbf{c}(z, t)$ can be reconstructed from the various extents by pre-multiplying (39) by $\mathcal{T}^{-1}=\left[\begin{array}{lll}\mathbf{N}^{\mathrm{T}} & \mathbf{E}_{d} & \mathbf{P}\end{array}\right]$ and considering the fact that $\mathbf{x}_{i v}(z, t)=\mathbf{0}_{q}$ :

$\delta \mathbf{c}(z, t)=\mathbf{N}^{\mathrm{T}} \mathbf{x}_{r}(z, t)+\mathbf{E}_{d} \mathbf{x}_{d}(z, t)$.

Finally, the concentrations $\mathbf{c}(z, t)$ are obtained from Eqs. (37) and (41) as:

$\mathbf{c}(z, t)=\mathbf{N}^{\mathrm{T}} \mathbf{x}_{r}(z, t)+\mathbf{E}_{d} \mathbf{x}_{d}(z, t)+\mathbf{c}_{i b c}(z, t)$.

\section{Remarks}

- The concentration of the $S$ species at a given position and time can be transformed into $R$ extents of reaction and $p_{d}$ extents of diffusion. The only requirement is $\operatorname{rank}\left(\left[\mathbf{N}^{\mathrm{T}} \mathbf{E}_{d}\right]\right)=R+p_{d}$, which implies that the number of diffusing species $p_{d}$ can be at most $S-R$.

- The linear transformation is independent of $z$ and $t$ and does not assume that the diffusivities of the species are equal. Furthermore, the transformation is valid for any profile of the convective velocity and of the initial and inlet concentrations.

A possible use of this decoupling regards the identification of reaction rates independent of diffusion, and the identification of diffusion rates independently of any kinetic information.

\section{Application of the decoupling transformation}

This section is not meant to discuss a specific case study but rather to guide the reader through potential uses of the concept of extents. The decoupling transformation can be used for two different classes of applications, namely, (i) to simplify the dynamic model, its analysis and possibly the design of model-based monitoring, estimation, control and optimization schemes, and (ii) to process measured data by either isolating certain signals that are useful for kinetic identification or reconstructing certain quantities in the absence of a kinetic model. These two classes of problems are briefly discussed next.

\subsection{Model-based applications}

- Model reduction. The reaction system can be described by either the $S$ numbers of moles $\mathbf{n}(t)$ given by the differential Eq. (1a) or the $(R+p+1)$ extents given by the differential Eqs. (13a)-(13c). The dimensionality of the system is therefore $d:=R+p+1$. However, note that the transformed model (13a)-(13c) is not a minimalstate representation of the system (1a) since the reaction rates 
$\mathbf{r}_{v}(t)=V(t) r(\mathbf{c}(t))$ cannot be computed solely from the reduced states $\mathbf{x}_{r}(t), \mathbf{x}_{i n}(t)$ and $x_{i c}(t)$ (Bhatt et al., 2012, 2010). Indeed, the description of the concentrations $\mathbf{c}(t)=\mathbf{n}(t) / V(t)$ requires the knowledge of $\mathbf{n}_{0}$, of dimension $S$, to reconstruct $\mathbf{n}(t)$ as per Eq. (14).

- State accessibility. The transformed system (13a)-(13d) indicates that $\mathbf{x}_{i v}$ is inaccessible. Hence, the maximal dimension of the accessible part is $R+p+1$. In other words, since $q$ needs to be zero for full state accessibility, there should be at least $S-R-1$ inlet streams. Note that this result, which has been reported previously in the literature (Bastin and Lévine, 1993), follows trivially from the decoupling transformation.

- Control. The idea is to work with the reduced model that does not include the inaccessible invariant part. With the inlet flows or the heat input as manipulated variables, the resulting models are typically input-affine and lend themselves well to control via feedback linearization, for which certain conditions are necessary (Fliess et al., 1995; Srinivasan et al., 1998). Applications of such control approaches are available in the literature (Farschman et al., 1998; Favache and Dochain, 2009; Hoang et al., 2014).

\subsection{Data-driven applications}

- Reconstruction of extent and rate profiles. With the knowledge of $\mathbf{N}$, $\mathbf{W}_{\text {in }}$ and $\mathbf{n}_{0}$, the transformation $\mathcal{T}$ allows computing the various extents at the time instant $t_{h}$ from the discrete measurements $\mathbf{n}\left(t_{h}\right)$. On the other hand, as expressed by Eq. (13a), the extent of reaction $x_{r, i}(t)$ is the output of a dynamic system whose input is the reaction rate $r_{v, i}(t)$. It follows that the rate profile $r_{v, i}\left(t_{h}\right)$ can be reconstructed from $x_{r, i}\left(t_{h}\right)$ by system inversion (Mhamdi and Marquardt, 2004).

- Kinetic identification. Kinetic identification is performed by comparing the rates or extents computed from measured concentrations to the values predicted by the model. This comparison can be done individually for each reaction (Bardow and Marquardt, 2004; Brendel et al., 2006; Michalik et al., 2009). This way, several rate expressions can be compared to experimental data, one at a time, until the correct expression has been found and the corresponding parameters identified. The route over extents has certain advantages, in particular in the presence of noisy and scarce measurements (see Appendix C) (Bhatt et al., 2011, 2012).

- State reconstruction. The objective is to reconstruct the state $\mathbf{n}\left(t_{h}\right)$ of dimension $S$ from $S_{a}<S$ measured species $\mathbf{n}_{a}\left(t_{h}\right)$. When all kinetic models are available and the model is observable, the unmeasured states can be reconstructed via dynamic reconstruction (state observers). Alternatively, when there are at least as many measured species as independent reactions, that is $S_{a} \geq R$, $\mathbf{n}\left(t_{h}\right)$ can be reconstructed without knowledge of kinetics (Billeter et al., 2013). This is a two-step procedure, whereby the extents are first estimated using either Eq. (9) or the approach described in Appendix C. The unmeasured species are then reconstructed from the estimated extents. For the case $S_{a}<R$, it is no longer possible to compute all extents of reaction from $\mathbf{n}_{a}\left(t_{h}\right)$, in which case only a part of $\mathbf{x}_{r}\left(t_{h}\right)$ is computed without a kinetic model, with the remaining extents being computed using a kinetic model.

\section{Conclusions}

The concept of reaction variants/invariants has been around for nearly 60 years. The reaction invariants, which are typically computed from the knowledge of stoichiometry (or, almost equivalently, from the atomic matrix) are not affected by the progress of the reactions. The reaction variants are often chosen orthogonal to the reaction invariants. Unfortunately, a reaction variant may also be affected by other phenomena such as flows and mass transfers. Similarly, although a reaction invariant is unaffected by reaction, it might sense the effect of flows and mass transfers. Hence, the applicability of the concept of reaction variants/invariants has been limited to specific reactor arrangements with negligible overlap of the reaction and transport phenomena.

This paper has addressed the computation of variant and invariant quantities for open both homogeneous and heterogeneous reaction systems. The concept of reaction variants/invariants that is used extensively in the context of batch processes has been extended to take into account the effects of inlet and outlet flows, of mass transfers between phases, and of convection and diffusion. In contrast to previously defined variants, each extent developed in this work describes uniquely and completely the progress of the corresponding process. Furthermore, the invariants defined in this work are true invariants that are identically equal to zero and can be discarded from the dynamic model.

Isolation of the individual phenomena is implemented via decoupling of the balance equations through linear transformation. The transformation uses structural information about the reaction system, in particular the stoichiometry, the inlet composition, the initial conditions, and the knowledge of the species that transfer between phases. If these parameters are constant, the transformation is globally valid and straightforward to implement. Otherwise, things are more complicated and one might need to rely on a piecewise transformation. For a convective and/or diffusive system, it is also necessary to know the convective velocity and/or the identity of the species that diffuse.

The significance of this work is twofold: (i) at the scientific level, the separation of reaction and transport phenomena has significant implications for kinetic identification (Bhatt et al., 2012), model reduction (Bhatt et al., 2012), state reconstruction (Bastin and Dochain, 1990; Bhatt, 2011) and leads to a better understanding of chemical reaction systems, and (ii) at the application level, a systematic procedure is available for developing kinetic models in the laboratory and design targeted monitoring, control and optimization schemes for production (Georgakis, 1986; Farschman et al., 1998; Hoang et al., 2014). Both aspects will improve process operation in the long run.

This paper summarizes the efforts that have been done in the last decade to extend the concept of reaction variants/invariants, originally defined for batch homogeneous reactors, to multi-phase reaction systems with inlets and outlets. Compared to previous work done by the same research group, this paper has introduced several novel elements, namely, the key concept of linear transformation, the extension of the approach to distributed reaction systems as well as the treatment of the initial conditions via the introduction of deviation variables. More efforts are needed to develop the theory further and, for example, make it applicable to other lumped or distributed reaction systems, such as heterogeneous catalytic reaction systems and reaction-absorption or reaction-distillation columns. Finally, it is expected that similar decoupling transformations can be found for a wider class of processing systems as long as the balance equations can be characterized by a well-defined structure as that of Eq. (1a).

\section{Acknowledgements}

The authors would like to acknowledge insightful discussions with Dr. Michael Amrhein and Dr. Balasubrahmanyan Srinivasan.

\section{Appendix A. Reaction system in deviation variables}

This appendix proposes another way to perform the decoupling for homogeneous reaction systems, which parallels the method 
used above for distributed reaction systems. The idea is to work with numbers of moles that represent deviations from the effect of the initial conditions, $\mathbf{n}_{i c}(t)$, as shown below. ${ }^{9}$

\section{Mole balance equations}

The right-hand side of Eq. (1a) has three contributions that are associated with the reactions, the inlets and the outlet. Since the corresponding terms $\mathbf{N}^{\mathrm{T}} \mathbf{r}_{v}(t), \mathbf{W}_{\text {in }} \mathbf{u}_{\text {in }}(t)$ and $\omega(t) \mathbf{n}(t)$ appear linearly, they satisfy the principle of superposition and each contribution can be computed independently of the others. We will next compute the effect at time $t$ of the initial conditions, that is, the part of the initial conditions that has not been removed through the outlet flow, by solving the differential equation

$$
\dot{\mathbf{n}}_{i c}(t)=-\omega(t) \mathbf{n}_{i c}(t), \quad \mathbf{n}_{i c}(0)=\mathbf{n}_{0},
$$

with $\mathbf{n}_{i c}(t)$ indicating the numbers of moles that are due uniquely to the non-zero initial conditions. Since each element of $\mathbf{n}_{i c}(t)$ has the same dynamics, $\mathbf{n}_{i c}(t)$ can be expressed as

$\mathbf{n}_{i c}(t)=\mathbf{n}_{0} x_{i c}(t)$,

with $x_{i c}(t)$ given by

$\dot{x}_{i c}(t)=-\omega(t) x_{i c}(t), \quad x_{i c}(0)=1$.

The extent of initial conditions $x_{i c}(t)$ is a scalar that varies between 1 and 0 and indicates the fraction of the initial conditions that is still present in the reactor at time $t$.

Upon writing the concentrations as deviations from the concentrations $\mathbf{n}_{i c}(t)$,

$\delta \mathbf{n}(t):=\mathbf{n}(t)-\mathbf{n}_{i c}(t)$,

Eq. (1a) becomes:

$\delta \dot{\mathbf{n}}(t)=\mathbf{N}^{\mathrm{T}} \mathbf{r}_{v}(t)+\mathbf{W}_{i n} \mathbf{u}_{i n}(t)-\omega(t) \delta \mathbf{n}(t), \quad \delta \mathbf{n}(0)=\mathbf{0}_{S}$.

Vessel extents and invariants

The right-hand side of Eq. (47) has three contributions that indicate the effects of the reactions, the inlets and the outlet, respectively.

\section{Decoupling transformation}

The linear transformation $\mathcal{T}=\left[\begin{array}{lll}\mathbf{N}^{\mathrm{T}} & \mathbf{W}_{\text {in }} & \mathbf{P}\end{array}\right]^{-1}$ transforms $\delta \mathbf{n}(t)$ into three parts, namely $\mathbf{x}_{r}(t)$ and $\mathbf{x}_{i n}(t)$ that are associated with the reactions and the inlets, and $\mathbf{x}_{i v}(t)$ that is orthogonal to the other extents and invariant as will be seen below:

$$
\left[\begin{array}{l}
\mathbf{x}_{r}(t) \\
\mathbf{x}_{i n}(t) \\
\mathbf{x}_{i v}(t)
\end{array}\right]=\left[\begin{array}{l}
\mathbf{R} \\
\mathbf{F} \\
\mathbf{P}^{+}
\end{array}\right] \delta \mathbf{n}(t)=\mathcal{T} \delta \mathbf{n}(t)
$$

where the $S \times q$ matrix $\mathbf{P}$ describes the $q$-dimensional null space of the matrix $\left[\begin{array}{ll}\mathbf{N}^{\mathrm{T}} & \mathbf{W}_{i n}\end{array}\right]^{\mathrm{T}}$, with $q=S-R-p$. Note that the invariant space corresponding to Eq. (8) is of lower dimension, $q=S-R-p-1$, as one dimension is needed to represent the contribution of the initial conditions.

\footnotetext{
${ }^{9}$ An alternative consists in working with numbers of moles that represent deviations from the initial conditions $\mathbf{n}_{0}$. However, this way, the formulation and the interpretation of $x_{i c}(t)$ is not as straightforward.
}

Vessel extents and invariants

If $\operatorname{rank}\left(\left[\mathbf{N}^{\mathrm{T}} \mathbf{W}_{i n}\right]\right)=R+p$, the linear transformation (48) brings the dynamic model (47) to:

$$
\begin{aligned}
& \dot{x}_{r, i}(t)=r_{v, i}(t)-\omega(t), x_{r, i}(t) \quad x_{r, i}(0)=0 \quad i=1, \ldots, R \\
& \dot{x}_{i n, j}(t)=u_{i n, j}(t)-\omega(t), x_{i n, j}(t) \quad x_{i n, j}(0)=0 \quad j=1, \ldots, p \\
& \mathbf{x}_{i v}(t)=\mathbf{0}_{q} \text {. }
\end{aligned}
$$

The numbers of moles $\delta \mathbf{n}(t)$ can be reconstructed from the various extents by pre-multiplying (48) by $\mathcal{T}^{-1}=\left[\begin{array}{llll}\mathbf{N}^{T} & \mathbf{W}_{\text {in }} & \mathbf{P}\end{array}\right]$ and considering the fact that $\mathbf{x}_{i v}(t)=\mathbf{0}_{q}$ :

$\delta \mathbf{n}(t)=\mathbf{N}^{\mathrm{T}} \mathbf{x}_{r}(t)+\mathbf{W}_{i n} \mathbf{x}_{i n}(t)$.

Finally, the numbers of moles $\mathbf{n}(t)$ are obtained from (50) with (46) and (44) as:

$\mathbf{n}(t)=\mathbf{N}^{\mathrm{T}} \mathbf{x}_{r}(t)+\mathbf{W}_{i n} \mathbf{x}_{i n}(t)+\mathbf{n}_{0} x_{i c}(t)$.

Similarly, the mass $m(t)$ can be reconstructed from $\mathbf{x}_{i n}(t)$ and $x_{i c}(t)$ as follows:

$m(t)=\mathbf{1}_{p}^{\mathrm{T}} \mathbf{x}_{i n}(t)+m_{0} x_{i c}(t)$.

\section{Appendix B. Danckwerts boundary conditions}

This appendix shows that the transformation in Section 5 still holds when the reaction-convection-diffusion system (35) is written with the following Danckwerts boundary conditions (Danckwerts, 1953):

$\mathbf{c}(0, t)=\mathbf{c}_{i n}(t)-\frac{1}{v(0, t)} \mathbf{E}_{d} \mathbf{J}(0, t)$,

$\mathbf{J}(l, t)=\mathbf{0}_{p_{d}}$,

for a tubular reactor ranging from $z=0$ to $z=l$, where $\mathbf{J}(z, t)$ is the $p_{d}$-dimensional vector of diffusion fluxes in $\mathrm{kmol} /\left(\mathrm{m}^{2} \mathrm{~s}\right)$. The partial differential Eq. (38) has now the boundary conditions $\delta \mathbf{c}(0, t)=$ $-(1 / v(0, t)) \mathbf{E}_{d} \mathbf{J}(0, t)$ and $\mathbf{J}(l, t)=\mathbf{0}_{p_{d}}$. The diffusion flux $\mathbf{J}(z, t)$ is unknown, but it can be computed from the diffusion rates as follows:

$\frac{\partial \mathbf{J}(z, t)}{\partial z}=-\boldsymbol{\phi}(z, t) \quad \mathbf{J}(l, t)=\mathbf{0}_{p_{d}}$.

If $\operatorname{rank}\left(\left[\mathbf{N}^{\mathrm{T}} \mathbf{E}_{d}\right]\right)=R+p_{d}$, the linear transformation (39) brings (38) with the corresponding Danckwerts boundary conditions to:

$$
\begin{array}{r}
\frac{\partial \mathbf{x}_{r}(z, t)}{\partial t}+\frac{\partial\left(v(z, t) \mathbf{x}_{r}(z, t)\right)}{\partial z}=\mathbf{r}(z, t), \\
\mathbf{x}_{r}(z, 0)=\mathbf{0}_{R}, \\
\mathbf{x}_{r}(0, t)=\mathbf{0}_{R} \\
\frac{\partial \mathbf{x}_{d}(z, t)}{\partial t}+\frac{\partial\left(v(z, t) \mathbf{x}_{d}(z, t)\right)}{\partial z}=\boldsymbol{\phi}(z, t), \quad \mathbf{x}_{d}(z, 0)=\mathbf{0}_{p_{d}}, \\
\mathbf{x}_{d}(0, t)=-\frac{1}{v(0, t)} \mathbf{J}(0, t)
\end{array}
$$

$\mathbf{x}_{i v}(z, t)=\mathbf{0}_{q}$,

which corresponds to (40a)-(40c) with different boundary conditions. The boundary condition $\mathbf{J}(0, t)$ is computed from $\mathbf{J}(l, t)=\mathbf{0}_{p_{d}}$ using the ordinary differential Eq. (54).

Note that the Danckwerts boundary conditions assume that there is a discontinuity in the value of the diffusion fluxes at $z=0$ (Pearson, 1959), which is clearly illustrated by the non-zero values of the extents $\mathbf{x}_{d}(0, t)$. A detailed discussion of the differences between the Danckwerts boundary conditions and alternative formulations for tubular reactors can be found elsewhere (Parulekar and Ramkrishna, 1984). 


\section{Appendix C. On isolating reaction contributions}

We present two different ways of eliminating the effect of the inlet and outlet flows in the measured numbers of moles, and we will compare these approaches with that based on the linear transformation. In particular, we are interested in reconstructing the rate $r_{v, i}(t)$, the batch extent $\xi_{i}(t)$ and the vessel extent $x_{r, i}(t)$.

Numbers of moles in reaction-variant form

Re-writting Eq. (8) as:

$$
\dot{\mathbf{n}}(t)-\mathbf{W}_{i n} \mathbf{u}_{i n}(t)-\mathbf{n}_{0} \delta(t)+\omega(t) \mathbf{n}(t)=\mathbf{N}^{\mathrm{T}} \mathbf{r}_{v}(t), \quad \mathbf{n}(0)=\mathbf{0}_{S},
$$

and integrating gives:

$$
\mathbf{n}(t)-\mathbf{W}_{i n} \int_{0}^{t} \mathbf{u}_{i n}(\tau) d \tau-\mathbf{n}_{0}+\int_{0}^{t} \omega(\tau) \mathbf{n}(\tau) d \tau=\mathbf{N}^{\mathrm{T}} \int_{0}^{t} \mathbf{r}_{v}(\tau) d \tau .
$$

Upon defining the numbers of moles in reaction-variant $(R V)$ form,

$\mathbf{n}^{R V}(t):=\mathbf{n}(t)-\mathbf{n}_{0}-\mathbf{W}_{\text {in }} \int_{0}^{t} \mathbf{u}_{\text {in }}(\tau) d \tau+\int_{0}^{t} \omega(\tau) \mathbf{n}(\tau) d \tau$,

and the batch extents of reaction,

$\boldsymbol{\xi}(t):=\int_{0}^{t} \mathbf{r}_{v}(\tau) d \tau$

Eq. (57) gives:

$\mathbf{n}^{R V}(t)=\mathbf{N}^{\mathrm{T}} \boldsymbol{\xi}(t)$

or in differential form,

$\dot{\mathbf{n}}^{R V}(t)=\mathbf{N}^{\mathrm{T}} \mathbf{r}_{v}(t), \quad \mathbf{n}^{R V}(0)=\mathbf{0}_{S}$.

Numbers of moles in vessel reaction-variant form

If the inlet and outlet flow rates $\mathbf{u}_{\text {in }}(t)$ and $u_{\text {out }}(t)$ are known, one can compute $\mathbf{x}_{i n}(t)$ and $x_{i c}(t)$ according to (16) and then use (14) to compute the contribution of the reactions, labeled the numbers of moles in vessel reaction-variant $(v R V)$ form, as follows:

$\mathbf{n}^{v R V}(t):=\mathbf{n}(t)-\mathbf{W}_{i n} \mathbf{x}_{i n}(t)-\mathbf{n}_{0} x_{i c}(t)$,

which leads to:

$\mathbf{n}^{v R V}(t)=\mathbf{N}^{\mathrm{T}} \mathbf{x}_{r}(t)$,

or in differential form,

$\dot{\mathbf{n}}^{v R V}(t)=\mathbf{N}^{\mathrm{T}} \mathbf{r}_{v}(t)-\omega(t) \mathbf{n}^{v R V}(t), \quad \mathbf{n}^{v R V}(0)=\mathbf{0}_{S}$.

Expression (63) is very useful for analysis purposes as will be seen later. Note that $\mathbf{n}^{v R V}(t) \neq \mathbf{n}^{R V}(t)$ in the presence of an outlet.

\section{Comparison with linear transformation}

A key feature of the linear transformation $\mathcal{T}$ is its ability to isolate and remove the effect of the inlet and outlet streams from the measured numbers of moles. Next, we will compare the experimental implications of computing $r_{v, i}(t)$ from $\dot{\mathbf{n}}^{R V}(t), \xi_{i}(t)$ from $\mathbf{n}^{R V}(t)$ and $x_{r, i}(t)$ via the linear transformation from either $\mathbf{n}(t)$ or $\mathbf{n}^{v R V}(t)$. It is assumed here that the measured numbers of moles $\mathbf{n}(t)$ are noisy and only available unfrequently at the time instants $t_{h}, h=1,2, \ldots$.

1 Computation of $r_{v, i}\left(t_{h}\right)$ from $\dot{\mathbf{n}}^{R V}\left(t_{h}\right)$. This is done by inversion of Eq. (61), with $\dot{\mathbf{n}}^{R V}\left(t_{h}\right)$ computed from Eq. (58) as:

$$
\dot{\mathbf{n}}^{R V}\left(t_{h}\right)=\dot{\mathbf{n}}\left(t_{h}\right)-\mathbf{W}_{i n} \mathbf{u}_{i n}\left(t_{h}\right)+\omega\left(t_{h}\right) \mathbf{n}\left(t_{h}\right) .
$$

One sees that this operation requires the differentiation of the noisy and scarce signal $\mathbf{n}\left(t_{h}\right)$.

2 Computation of $\xi_{i}\left(t_{h}\right)$ from $\mathbf{n}^{R V}\left(t_{h}\right)$. This is done by inversion of Eq. (60), with $\mathbf{n}^{R V}\left(t_{h}\right)$ computed from Eq. (58). One sees that this operation requires the integration of the noisy and scarce signal $\mathbf{n}\left(t_{h}\right)$.

3 Computation of $x_{r, i}\left(t_{h}\right)$ via linear transformation from $\mathbf{n}\left(t_{h}\right)$. This is done via Eq. (9) as $x_{r, i}\left(t_{h}\right)=\mathbf{R}_{i} \mathbf{n}\left(t_{h}\right)$, which does not require differentiation nor integration of noisy signals. The transformation requires at least $R+p+1$ measured species.

4 Computation of $x_{r, i}\left(t_{h}\right)$ via linear transformation from $\mathbf{n}^{v R V}\left(t_{h}\right)$. This is done via Eq. (63) as $x_{r, i}\left(t_{h}\right)=\left(\mathbf{N}^{\mathrm{T}^{+}}\right)_{i} \mathbf{n}^{v R V}\left(t_{h}\right)$, which does not require differentiation nor integration of noisy signals either. The transformation requires only $R$ measured species.

This shows that the path over the linear transformation, in particular using $\mathbf{n}^{v R V}\left(t_{h}\right)$ and Eq. (63), has a clear experimental advantage.

\section{References}

Aggarwal M, Balaji S, Ydstie BE. Invariant based modeling and control of multi-phase reactor systems. J Process Control 2011;21:1390-406.

Amrhein M, Bhatt N, Srinivasan B, Bonvin D. Extents of reaction and flow for homogeneous reaction systems with inlet and outlet streams. AIChE J 2010;56:2873.

Amrhein M. Reaction and flow variants/invariants for the analysis of chemical reaction data. Switzerland: EPFL Lausanne; 1998, Doctoral thesis No. 1861.

Asbjørnsen OA, Fjeld M. Response modes of continuous stirred tank reactors. Chem Eng Sci 1970;25:1627-36.

Asbjørnsen OA. Reaction invariants in the control of continuous chemical reactors. Chem Eng Sci 1972;27:709-17.

Bardow A, Marquardt W. Incremental and simultaneous identification of reaction kinetics: Methods and comparison. Chem Eng Sci 2004;59(13):2673.

Bastin G, Dochain D. On-line estimation and adaptive control of bioreactors. Elsevier: Amsterdam; 1990.

Bastin G, Lévine J. On state accessibility in reaction systems. IEEE Trans Autom Control 1993;38(5):733-42.

Bhatt N, Amrhein M, Bonvin D. Minimal state representation for homogeneous reaction systems. In: ESCAPE-20; 2010.

Bhatt N, Amrhein M, Bonvin D. Extents of reaction, mass transfer and flow for gasliquid reaction systems. Ind Eng Chem Res 2010;49:7704.

Bhatt N, Amrhein M, Bonvin D. Incremental identification of reaction and mass-transfer kinetics using the concept of extents. Ind Eng Chem Res 2011;50(23):12960-74.

Bhatt N, Amrhein M, Srinivasan B, Mullhaupt Ph, Bonvin D. Minimal state representation for open fluid-fluid reaction systems. In American Control Conference. Canada: Montreal; 2012.

Bhatt N, Kerimoglu N, Amrhein M, Marquardt W, Bonvin D. Incremental identification for reaction systems - a comparison between rate-based and extent-based approaches. Chem Eng Sci 2012;83:24-38

Bhatt NP. Extents of reaction and mass transfer in the analysis of chemical reaction systems. Switzerland: EPFL Lausanne; 2011, Doctoral thesis No. 5028.

Billeter J, Srinivasan S, Bonvin D. Extent-based kinetic identification using spectroscopic measurements and multivariate calibration. Anal Chim Acta 2013;767:21-34.

Bonvin D, Rippin DWT. Target factor analysis for the identification of stoichiometric models. Chem Eng Sci 1990;45(12):3417-26.

Brendel M, Bonvin D, Marquardt W. Incremental identification of kinetic models for homogeneous reaction systems. Chem Eng Sci 2006;61(16):5404-20.

Danckwerts PV. Continuous flow systems: distribution of residence times. Chem Eng Sci 1953;2(1):1-13.

Dochain D, Couenne F, Jallut C. Enthalpy based modelling and design of asymptotic observers for chemical reactors. Int J Control 2009;82(8):1389-403.

Fan LT, Ahn YK. Critical evaluation of boundary conditions for tubular flow reactors. Ind Eng Chem Process Des Dev 1962;1(3):190-5.

Farschman CA, Viswanath KP, Ydstie BE. Process systems and inventory control. AIChE J 1998;44(8):1841-57.

Favache A, Dochain D. Thermodynamics and chemical systems stability: the CSTR case study revisited. J Process Control 2009;19(3):371-9.

Fjeld M, Asbjørnsen OA, Åström KJ. Reaction invariants and their importance in the analysis of eigenvectors, state observability and controllability of the continuous stirred tank reactor. Chem Eng Sci 1974;29:1917-26.

Fliess M, Lévine J, Rouchon P. Flatness and defect of nonlinear systems: introductory theory and examples. Int J Control 1995;61:1327-61.

Friedly JC. Dynamic behavior of processes. Englewood Cliffs, NJ: Prentice-Hall; 1972

Friedly JC. Extent of reaction in open systems with multiple heterogeneous reactions. AIChE J 1991;37(5):687-93.

Friedly JC. Reaction coordinates for heterogeneous flow reactors: physical interpretation. AIChE J 1996;42(10):2987-9. 
Gadewar SB, Doherty MF, Malone MF. A systematic method for reaction invariants and mole balances for complex chemistries. Comput Chem Eng 2001;25(9-10):1199-217.

Gadewar SB, Doherty MF, Malone MF. Reaction invariants and mole balances for plant complexes. Ind Eng Chem Res 2002;41:3771-83.

Georgakis C. On the use of extensive variables in process dynamics and control. Chem Eng Sci 1986;41:1471-84.

Grüner S, Mangold M, Kienle A. Dynamics of reaction separation processes in the limit of chemical equilibrium. AIChE J 2006;52:1010-26.

Hammarstrom LG. Control of chemical reactors in the subspace of reaction and control variants. Chem Eng Sci 1979;34:891-9.

Hoang NH, Dochain D, Ydstie BE. Partial inventory control of the CSTR via reactiondependent generalized inventories. In: 19th IFAC World Congress; 2014.

Marquardt W. Model-based experimental analysis: from experimental data to mechanistic models of kinetic phenomena in reactive systems. Chem Eng Sci 2008;63:4637-9.

Mhamdi A, Marquardt W. Estimation of reaction rates by nonlinear system inversion. In: ADCHEM 2003; 2004. p. 171-6.
Michalik C, Brendel M, Marquardt W. Incremental identification of fluid multi-phase reaction systems. AIChE J 2009;55:1009-22.

Parulekar SJ, Ramkrishna D. Tubular reactor stability revisited without the Danckwerts boundary conditions. Chem Eng Sci 1984;39(3):455-69.

Pearson JRA. A note on the "Danckwerts" boundary conditions for continuous flow reactors. Chem Eng Sci 1959;10(4):281-4.

Schneider DR, Reklaitis GV. On material balances for chemically reacting systems. Chem Eng Sci 1975;30:243-7.

Srinivasan B, Amrhein M, Bonvin D. Reaction and flow variants/invariants in chemical reaction systems with inlet and outlet streams. AIChE J 1998;44(8): 1858-67.

Srinivasan S, Billeter J, Bonvin D. Extent-based incremental identification of reaction systems using concentration and calorimetric measurements. Chem Eng J 2012;207-208:785-93.

Waller KV, Mäkilä PM. Chemical reaction invariants and variants and their use in reactor modeling, simulation, and control. Ind Eng Chem Process Des Dev $1981 ; 20: 1-11$. 